\title{
A Density Control Scheme Based on Disjoint Wakeup Scheduling in Wireless Sensor Networks
}

\author{
EunHwa Kim \\ Graduate School of Education, Yongin University, Korea \\ ehkimanna@yongin.ac.kr
}

\begin{abstract}
Since sensor nodes have limited battery power, it is desirable to select a minimum set of working sensor nodes which should be involved in sensing and forwarding data to the sink. In this paper, we propose a disjoint scheduling for density control in wireless sensor network to achieve the desired robust coverage as well as satisfactory connectivity to the sink with a small set of working nodes. Simulations showed that our scheme works well in an energy efficient fashion by turning off too many redundant nodes.
\end{abstract}

Keywords: density control, energy efficient, wireless sensor network

\section{Introduction}

Advanced sensor and wireless communications technologies have enabled the development of wireless sensor networks which can be used for various applications such as earth-quake, forest fire, battlefield surveillance, machine failure diagnosis, biological detection, home security, smart spaces and inventory tracking [1, 2].

A wireless sensor network consists of various types of sensor nodes, which is capable of detecting some phenomena, and sink node. The sensor node has a variety of types of sensors, data processor and wireless communication interfaces, which enable it to gather environmental information. Also, the sensor node generates and delivers messages to sink node [3]. The sink node aggregates, analyzes received messages, decides whether there are abnormal event occurred in the monitored area. The sink node also disseminates query messages to whole sensing field or specified field to get its concerned data.

In a multi-hop ad hoc sensor network, each node plays as data originator and data router. The failure of few nodes can cause significant topological changes and might require packet re-routing and network re-organization. Hence, power management becomes more important and many researchers are currently focusing on the design of energy efficient protocols and algorithms for sensor networks.

Power consumption is not the primary consideration in case of wireless mobile networks because power resources can be recharged by the user. This network request better quality of service (QoS) than the power efficiency. However power efficiency is an important performance metric in sensor networks that directly influences the network lifetime [4-6]. Application specific protocols can be designed appropriately by trading off other performance metrics such as delay and throughput with power efficiency.

In a large-scale sensor network, we need to deploy many sensor nodes closely together in the sensing field and maintain them in a proper way. One of the most important issues in such dense sensor networks is density control [7], which keeps distribution density of the working sensors at certain level [8]. Density control schemes select a set of working nodes to satisfy 
the coverage as well as the connectivity. It can save energy by turning off redundant nodes and can prolong the system lifetime by replacing the failed nodes with some sleeping nodes.

Scheduling scheme at sensor node is a very important factor related to energy efficiency in topology control of wireless sensor networks. In this paper, we propose an energy efficient scheduling scheme to satisfy requirements for sensing coverage and network connectivity. We select a small subset of working nodes in the deployed nodes to provide full coverage and connectivity to the sink node by a hop-based disjoint wakeup scheduling.

The rest of this paper is organized as follows. In Section 2, we introduce related works such as GAF, OGDC, ASCENT and Joint Scheduling. In Section 3, we address the hop-based disjoint wakeup scheme and we evaluate the proposed algorithm through simulation in Section 4, and finally conclude this paper in Section 5.

\section{Related Works}

In a wireless sensor network, saving energy is a major design factor because it is difficult to recharge a battery. Many methods are proposed to minimize energy consumption and prolong the lifetime of sensor network. In this section, we give a summary of some related works.

H. Zhang and J. C. Hou focus only on the coverage problems since they prove that if the transmission radius is at least twice of the sensing radius, a complete coverage of a convex area implies connectivity among the working set of sensor nodes [8]. They propose a completely localized density control algorithm, called OGDC [8]. This algorithm selects sensor nodes, which are close to optimal locations, to be active.

Some protocols suggest scheduling scheme with accurate location information of sensor nodes. In GAF, total network is divided into a smaller virtual grid cell enough to communicate with its neighbors and one sensor node is selected as active node in a virtual grid cell [9]. This method provides full coverage and connectivity but it needs the accurate information of sensor nodes and its neighbor nodes.

OGDC proves that if transmission radius is greater than twice of sensing radius, the full coverage provides guaranteed connectivity automatically. Initially some sensor nodes are randomly waked up. Then, they choose their neighbors within a distance of $\sqrt{3}$ (sense_radius), and the next active node are selected by the closest two nodes.

Since it is usually expensive to obtain and maintain the location information of each sensor node, some protocols do not use it. In ASCENT, sensor node decides whether or not to participate in configuration of the network topology based on some parameters such as the number of neighbors and packet loss ratio [10].

Joint scheduling offers a satisfactory coverage ratio by awaking sensor nodes at a random time slot. It wakes up at the other extra time slot which is allocated to its downstream hop neighbors to guarantee the connectivity to the sink node [8]. It was shown that this kind of random awake method is proper to solve the coverage problem [12-14]. To provide connection to the sink node certainly, every node checks if there is one more active upstreamhop nodes with the same time slot. 


\section{Hop-based Disjoint Wakeup Scheduling}

\subsection{Basic Concept}

In this section, we introduce a hop-based disjoint wakeup scheduling to preserve network coverage and connectivity with a small number of sensor nodes in wireless sensor networks. In our method, we assume wireless sensor network has the following conditions;

- sensor nodes are homogeneous, energy constrained and stationary,

- sensing and radio transmission radius of a sensor node are the same,

- sensor nodes are arbitrarily located densely,

- sensor node knows its one-hop neighbor’s information using hello messages,

- sensor node does not have accurate position information, and

- sensor nodes are synchronized to the standard time.

Active sensor nodes keep to wake up continuously and relay data packet while other sensor nodes remain in power-saving mode and periodically check if they can wake up to become active. Our scheme aims at both a perfect coverage ratio and a full connectivity to the sink node with a small number of active nodes in densely deployed networks as depicted in Figure 1. To obtain a perfect coverage, a sensor node should wake up if there is no active neighbor within its transmission radius. To get a full connectivity, an active sensor node must have at least one active upstream neighbor. In our scheme, each active node selects one of its upstream neighbors when there is no active upstream neighbor (HDS-SU). This ensures that every sensor node can have at least one upstream neighbor.

neighbor node: a sensor node within its transmission radius.

upstream neighbor: a neighbor node with a smaller hop count by one than itself.

downstream neighbor: a neighbor node with a larger hop count by one than itself.

peer neighbor: a neighbor node with the same hop count.

adjacent node: a sensor node sharing its area within transmission radius.

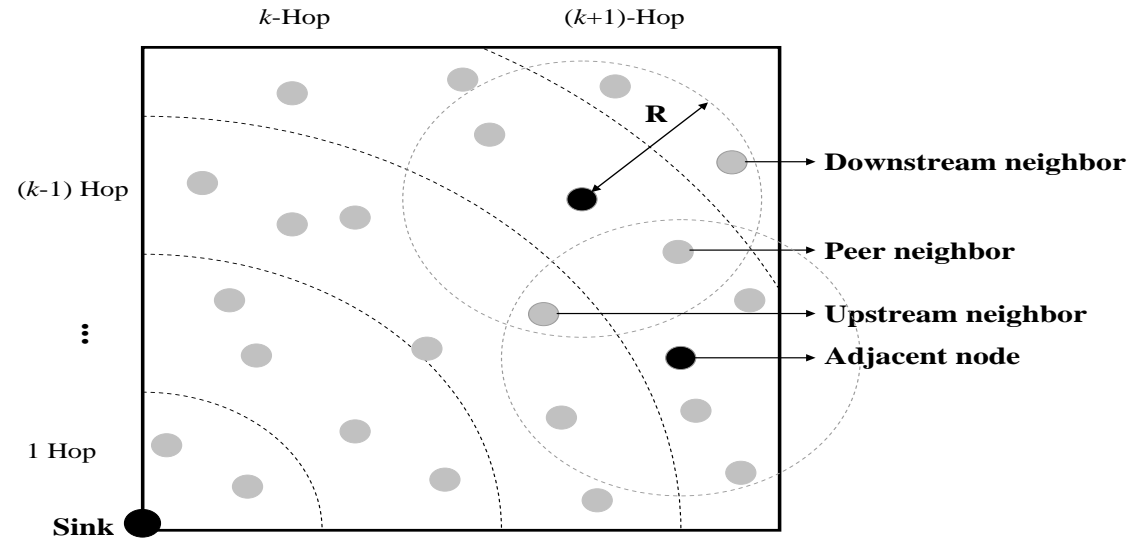

Figure 1. Types of Neighbors in Hop-based Sensor Networks 


\subsection{Detailed Operation}

Every active sensor node periodically broadcasts a HELLO_MSG, by which each sensor node constructs a list of its neighbors. Based on this local information, each node determines its activation. Our scheduling scheme uses three qualifications and three announcements as follows.

active_qualification_1: if none of its peer neighbors are active. If this condition is met, a sensor node should be active.

active_qualification_2: if it receives a SELECT_MSG, which is sent by an active sensor node when it does not have active upstream neighbors. If this condition is met, a sensor node should be additionally active

sleep_qualification: if one of its peer neighbors is active and it is not required as active upstream neighbor to all of its downstream neighbors. If this condition is met, a sensor node goes to sleep state.

Figure 2 shows an example, in which a sensor node satisfying active_qualification_1 wakes up and other sensor nodes become active additionally by active_qualification_2.

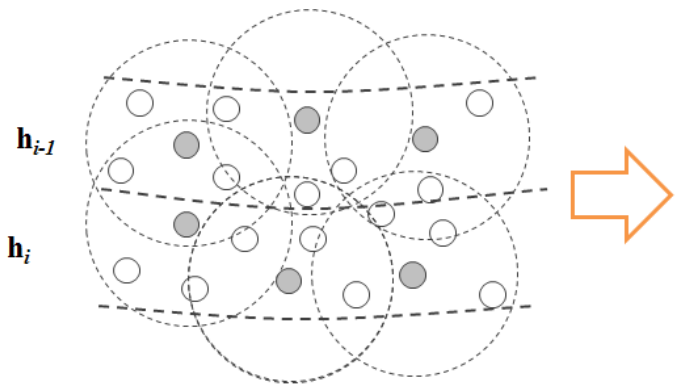

Active qualification_1

Sleep nodes

Active nodes

Additional Active nodes

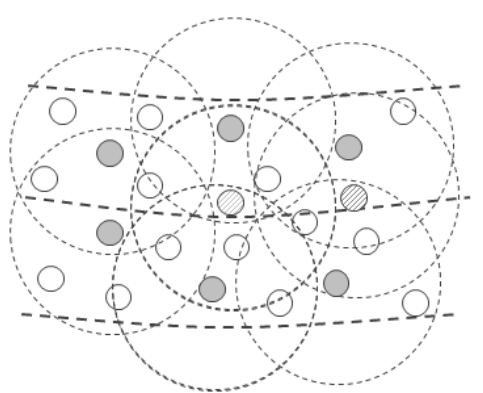

Active qualification_2

$\mathbf{h}_{i}$ : minimum hop count

Figure 2. An Example Showing Activation of Sensor Nodes

\section{Active Announcement_1}

Periodically, a sleeping node switches its state to LISTEN, and it turns on its radio and checks if it should become active or not. At this time, a sensor node switches its state to ACTIVE if the following two conditions are met. First, a sensor node should become active if the active_qualification_1 is satisfied. If several sensor nodes justify active_qualification_1 at the same time, there happens an announcement contention. In this case, we resolve the contention by using a randomized back-off delay. At the end of the back-off time, it checks the active_qualification_1 again, and it changes its state to ACTIVE if and only if the condition is satisfied. The back-off delay is given by

$$
\text { del ay }=\left(\left(1-\frac{E_{r}}{E_{m}}\right)+\left(1-\frac{C_{i}}{N_{i}}\right)+v\right) \times N_{i} \times T
$$

where the meaning of symbols are listed in Table 1. 
Table 1. Symbols used in Calculation of the Backoff Time

\begin{tabular}{|c|l|}
\hline Symbol & \multicolumn{1}{c|}{ Description } \\
\hline$N_{i}$ & the number of neighbor for sensor node i \\
\hline$C_{i}$ & the number of active upstream neighbors \\
\hline$E_{m}$ & the maximum energy available at a node \\
\hline$E_{r}$ & the remaining energy at a node \\
\hline$V$ & random variables uniformly selected from interval $(0,1]$ \\
\hline$T$ & the round-trip delay over the wireless link \\
\hline
\end{tabular}

Second, a sensor node in LISTEN state switches to ACTIVE after $t_{L}$ which means the maximum time allowed to stay at LISTEN state. To elect more active sensor nodes when a sensor node loses HELLO_MSGs, $t_{L}$ is given by

$$
t_{L}=3 \times N_{i} \times T
$$

which is the maximum value of back-off time determined by equation (1).

Unless a sensor node does not justify the sleep_qualification, it switches to ACTIVE state after this time period. Figure 3 shows the procedure of active announcement.

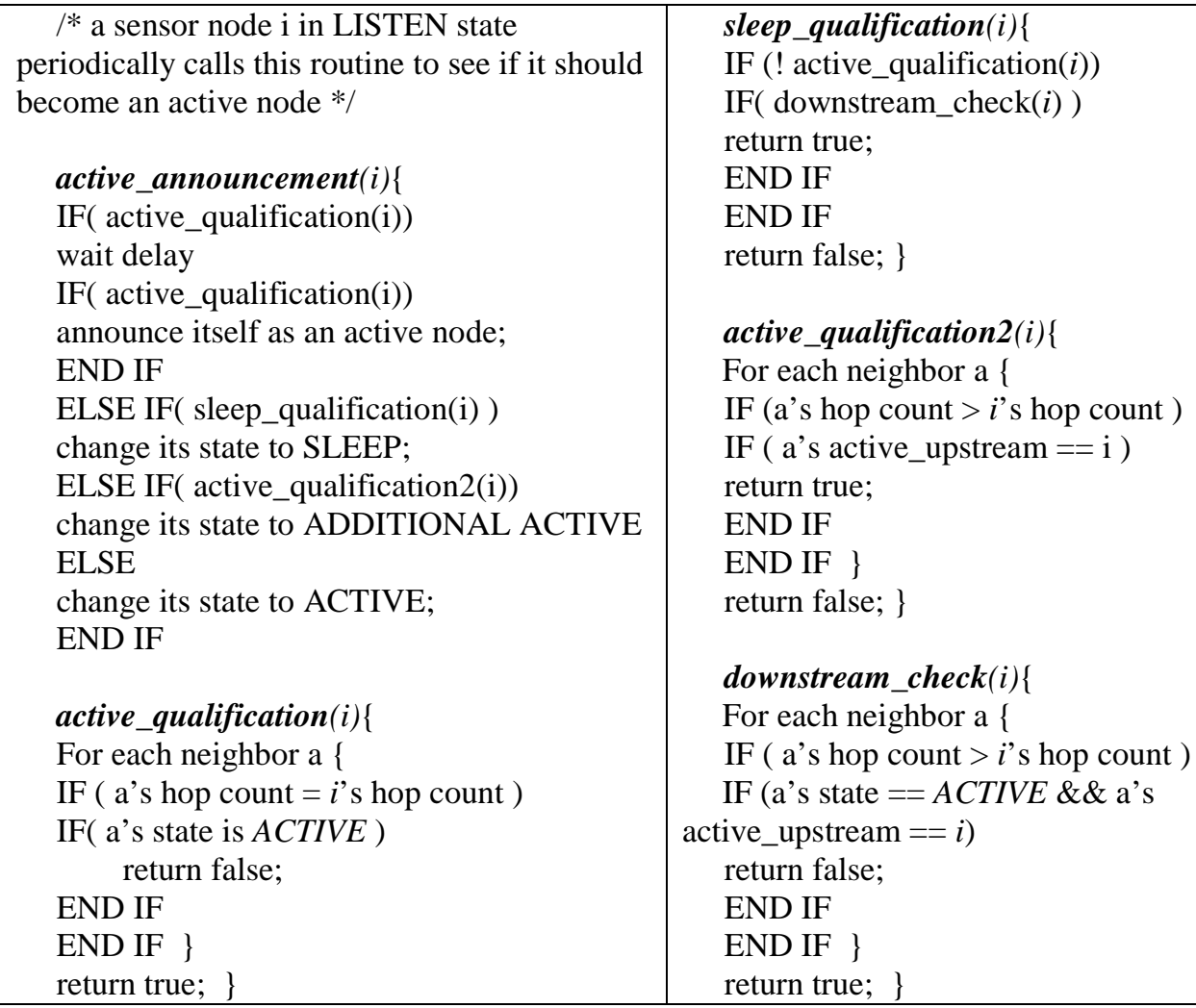

Figure 3. Procedure for Active Announcement 


\section{Active Announcement_2}

After switching to ACTIVE state, the sensor node checks if there are active upstream neighbors. If not, the active sensor node unicasts a SELECT_MSG to one of its upstream neighbors.

To select sensor nodes with much remaining energy, the sensor node stores the activation count of neighbors by HELLO_MSG. In LISTEN state, a sensor node receiving a SELECT_MSG switches its state to ADDITIONAL_ACTIVE.

If upstream neighbor fails due to some physical damage or environmental interference, the active sensor node broadcasts HELLO_MSG to make a peer neighbor take part as a relaying node. A peer neighbor that has active neighbors sends an ACK_MSG, and the active sensor node selects one of them, which has the most remaining energy, as a relaying node.

\section{Withdrawal Announcement}

To give each node a fair chance of being selected as active node, each node can stay in $A C T I V E$ for a certain time period $t_{A}$ at maximum. This time period $t_{A}$ is determined depending on the remaining energy at each node. An active sensor node periodically checks its remaining energy, and it changes its state to WITHDRAWAL when its remaining energy reaches some threshold value. The withdrawing neighbor is treated as a sleeping node during the time period $t_{L}$. After $t_{L}$, the sensor node checks its sleep_qualification again. And, if sleep_qualification is satisfied, it goes to SLEEP. Otherwise, it changes back to its previous state. Figure 4 shows the state transition diagram for hop-based disjoint wakeup scheduling.

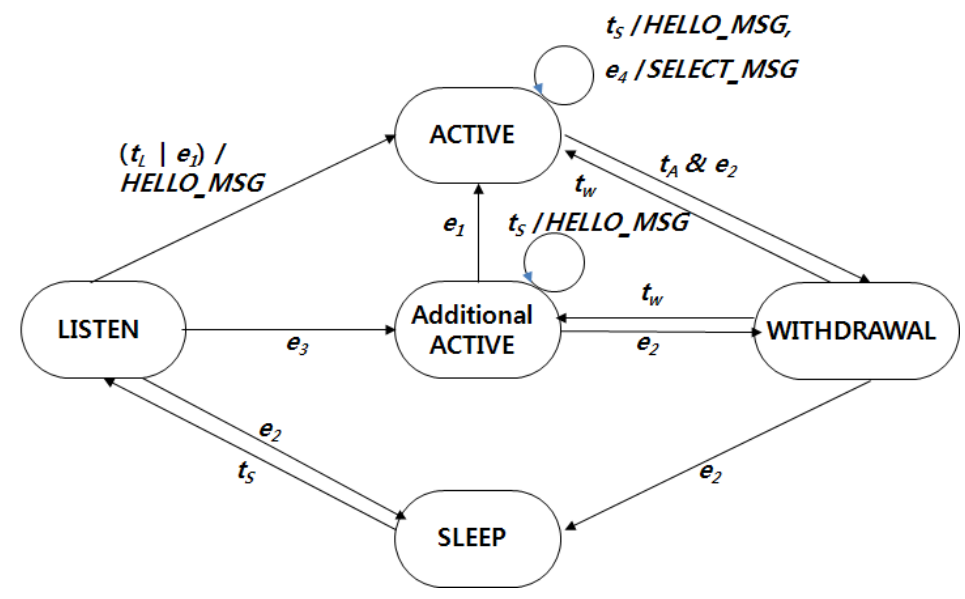

(a) Status transition diagram of sensor node

\begin{tabular}{|c|c|c|l|}
\hline \multirow{2}{*}{ State } & \multicolumn{3}{|c|}{ Description } \\
\cline { 2 - 4 } & Radio & Communication & \multicolumn{1}{c|}{ Characteristic } \\
\hline LISTEN & On & Receive only & Receive broadcast msg. \\
\hline ACTIVE & On & Receive/transmit & \\
\hline Additional ACTIVE & On & Receive/transmit & Relaying node \\
\hline WITHDRAWAL & On & Receive/transmit & For energy distribution \\
\hline SLEEP & Off & $\mathrm{X}$ & Power saving state \\
\hline
\end{tabular}

(b) State table 


\begin{tabular}{|c|l|}
\hline Event & \multicolumn{1}{c|}{ Description } \\
\hline$e_{1}$ & is satisfied active qualification_1 after random back_off \\
\hline$e_{2}$ & is satisfied sleep qualification \\
\hline$e_{3}$ & is satisfied active qualification_2 \\
\hline$e_{4}$ & no active upstream neighbors \\
\hline$t_{L}$ & the scheduled time period of $L I S T E N$ state \\
\hline$t_{A}$ & the scheduled time period of $A C T I V E$ state \\
\hline$t_{S}$ & the scheduled time period of SLEEP state \\
\hline$t_{W}$ & the scheduled time period of $W I T H D R A W A L$ state \\
\hline
\end{tabular}

\begin{tabular}{|c|l|}
\hline Action & \multicolumn{1}{|c|}{ Description } \\
\hline$H E L L O \_M S G$ & Broadcast HELLO_MSG \\
\hline SELECT_MSG & Unicast SELECT_MSG to a specific neighbor \\
\hline
\end{tabular}

(c) Event and action table

Figure 4. Status Transition Diagram of Sensor Node

\section{Performance Evaluation}

In this section, we report results from experiments to measure the ratio of coverage and connectivity of the network. Experiments are carried out on a simulator implemented in C/Java on the top of the 802.11 MAC ad hoc power-saving mode in SPAN [15].

\subsection{Simulation Environments}

To evaluate the proposed scheme, we deploy sensor nodes over a square region in a uniformly random fashion. The sink node is located at the corner of the sensing field. We assume that

- sensing field is flat region with a rectangular shape,

- each sensor node has a flat disk shape with sensing and transmission radius,

- there is a symmetric link between sensor nodes,

- the link is error free, and

- the traffic load is so light that link failure is mainly caused by network partition.

\subsection{Simulation Results}

The Ratio of Network Coverage

It is defined as the ratio of the area covered by active sensor nodes to the whole network area. A high ratio of network coverage means high sensing quality. We calculate the ratio of network coverage by counting the number of units detected by active sensor nodes over the total number of units within the sensing field.

Figure 5(a) shows the coverage ratio of GAF, Joint Scheduling and HDS-SU as we vary the density of deployed nodes, denoted by

$$
\rho=\frac{\# \text { of sensor nodes }}{\text { transmission r adi us }}=\frac{N * \pi * R^{2}}{A}
$$


where $N, A$, and $R$ denote the total number of deployed sensor nodes, the area of the whole sensing field, and the transmission radius of sensor node, respectively.

In Joint Scheduling, the number of random periods is critical parameter. We use a proper parameter calculated in [11].

Figure 5(a) indicates that three schemes can fully cover the whole network field when there are more than about 8 nodes deployed in the transmission range. Figure 5(b) shows that the disjoint wakeup scheduling uses a smaller number of active nodes than GAF and Joint Scheduling to obtain full coverage. This means that the disjoint wakeup scheduling offers a better coverage performance than the other two schemes with the same number of active nodes in a dense network.

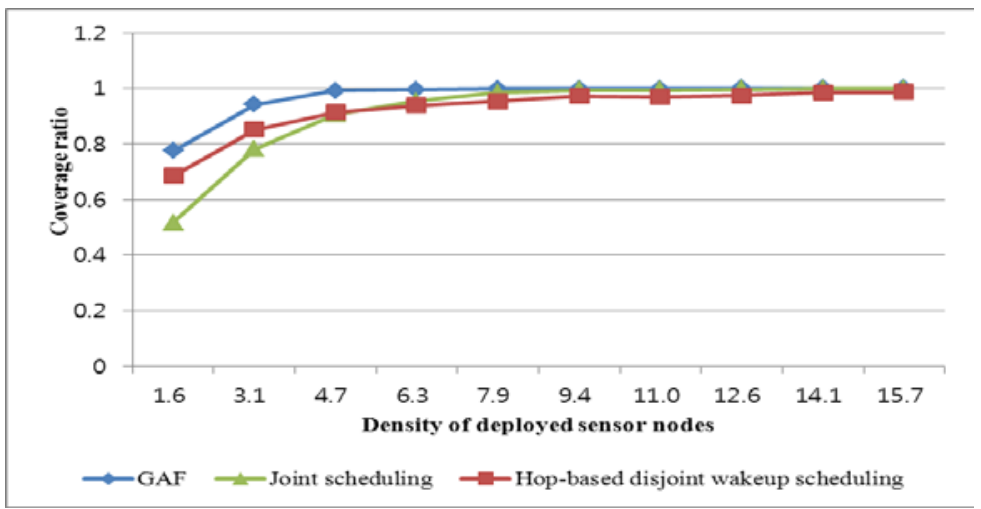

(a) Coverage ratio

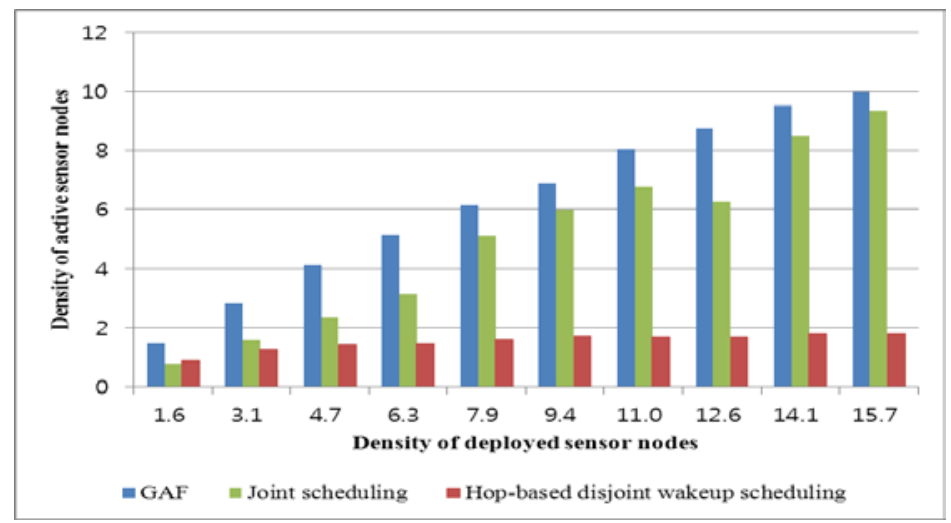

(b) Density of active sensor nodes

Figure 5. Coverage Ratio and Density of Active Sensor Nodes

\section{The Ratio of Network Connectivity}

It is defined as the ratio of the number of active nodes that have more than one active upstream neighbor, to the total number of active nodes. A higher ratio of network connectivity means that data can be delivered to the sink node with a higher possibility.

In Figure 6, we can see that all three schemes can guarantee connectivity in a dense network. However, GAF and Joint Scheduling produce more redundant active nodes than proposed method, as shown in Figure 5(b). 


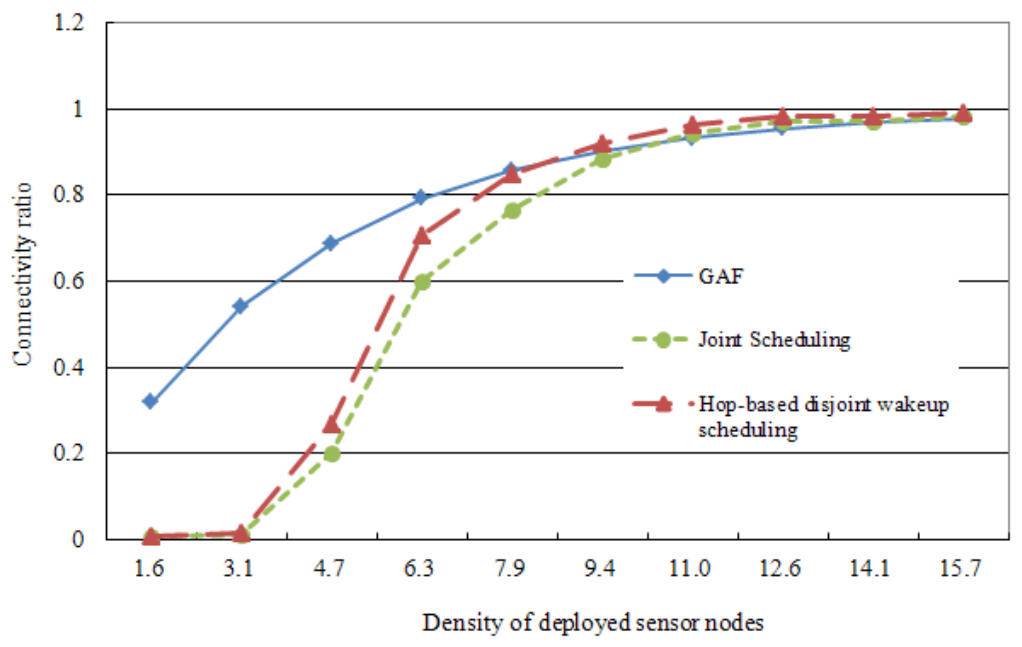

\section{Figure 6. Ratio of Network Connectivity as the Density of Deployed Sensor Nodes}

\section{Conclusion}

In this paper, we propose a density control scheme to avoid wasting excessive energy by turning off too many redundant nodes in wireless sensor network field. The proposed scheme can provide good network coverage and satisfactory network connectivity with a small set of active nodes by a hop-based disjoint wakeup scheduling. This scheme works in a distributed manner at each sensor node and automatically provides a routing path to the sink.

Simulation results show that the proposed scheme achieves the desired robust coverage as well as satisfactory connectivity to the sink node with a smaller number of active sensor nodes compared with GAF and Joint Scheduling. It is also shown that the proposed scheme works in a more energy-efficient fashion, so the network lifetime can be prolonged.

\section{References}

[1] F. Akyildiz, W. Su, Y. Sankarasubramaniam, and E. Cayirci, "Wireless Sensor Networks: A Survey”, Computer Networks, (2002) March, vol. 38, pp. 393-422.

[2] C. Chong and S. P. Kumar, "Sensor Networks: Evolution, Opportunities, and Challenges", Proceedings of the IEEE, vol. 91, (2003) August, pp. 1247-1256.

[3] D. Tian and N. D. Georganas, "A coverage-preserving node scheduling scheme for large wireless sensor networks”, Proceedings of ACM Wireless Sensor Network and Application Workshop, (2002) September, pp. 32-41.

[4] Zair Hussain, M. P. Singh and R. K. Singh, "Analysis of Lifetime of Wireless Sensor Network”, International Journal of Advanced Science and Technology, (2013) April , vol. 53, pp. 117-126.

[5] Razieh Sheikhpour, Sam Jabbehdari and Ahmad khademzadeh, "A Cluster-Chain based Routing Protocol for Balancing Energy Consumption in Wireless Sensor Networks”, International Journal of Multimedia and Ubiquitous Engineering, (2012) April, vol.7, no.2, pp. 1-16

[6] Choon-Sung Nam, Kyung-Soo Jang, Gyoo-Seok Choi and Dong-Ryeol Shin, "Study on Use of a Clustering Technique with Zone-Based Multi-hop Communication in Wireless Sensor Networks”, International Journal of Smart Home, (2012) April, vol. 6, no.2, pp. 65-70.

[7] Fan Ye, Gary Zhong, Songwu Lu, Lixia Zhang, "PEAS: A Robust Energy Conserving Protocol for LongLived Sensor Networks”, Proceedings of IEEE Network Protocols, (2002) November, pp. 200-201.

[8] H. Zhang and J. C. Hou, "Maintaining Sensing Coverage and Connectivity in Large Sensor Networks", Technical Report UIUC, UIUCDCS-R-2003-2351 (2003) 
[9] Y. Xu, S. Bien, Y. Mori, and J. Heidemann, “Topology Control Protocols to Conserve Energy in Wireless Ad Hoc Networks”, CENS Technical Report 0006, (2003)

[10] A. Cerpa and D. Estrin, "ASCENT: Adaptive self-configuring sensor networks topologies", IEEE Transactions on Mobile Computing, (2004) July, vol. 3, pp. 272-285.

[11] C. Liu, K. Wu, Y. Xiao, and B. Sun, "Random Coverage with Guaranteed Connectivity: Joint Scheduling for Wireless Sensor Networks”, IEEE Transactions on Parallel and Distributed Systems, (2006) June, vol. 17, pp. 562-575.

[12] Z. Abrams, A. Goel, and S. Plotkin, "Set k-cover algorithms for energy efficient monitoring in wireless sensor networks”, Proceedings of International Workshop on Information Processing in Sensor Networks, (2004) April, pp. 424-432.

[13] C. Liu, K. Wu, and V. King, "Randomized Coverage-Preserving Scheduling Schemes for Wireless Sensor Networks”, Proceedings of International IFIP-TC6 Networking Conference, (2005) May, vol. 3462.

[14] S. Slijepcevic and M. Potkonjak, "Power efficient organization of wireless sensor networks", Proceedings of IEEE International Conference on Communications, (2001) June, vol. 2, pp. 471-476.

[15] B. Chen, K. Jamieson, H. Balakrishnan, and R. Morris, "Span: An Energy-Efficient Coordination Algorithm for Topology Maintenance in Ad Hoc Wireless Networks", Proceedings of International Conference on Mobile Computing and Networking, (2001) July, vol. 8, pp. 85-96.

\section{Author}

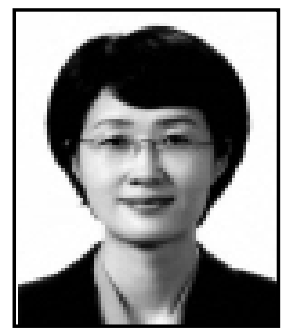

EunHwa Kim, BS degree in Computer Engineering at Kyoungpook National University in 1995. MS in Computer Engineering at Kyoungpook National University in 1999. Ph.D Computer Engineering at Kyoungpook National University in 2008. Software engineer at Samsung Eletronics from 1995 to 1996. Assistant Professor of the graduate school of education at Yongin University from 2012. 